\title{
Review of: "Evaluating the feasibility and acceptability of a community dialogue intervention in the prevention and control of schistosomiasis in Nampula province, Mozambique"
}

\section{Cheryl M E McCrindle}

Potential competing interests: The author(s) declared that no potential competing interests exist.

This is a very interesting piece of research. However, the publication is extremely long winded, more in the style of a thesis or textbook, than an article. In my opinion the discussion and conclusions were important. They indicated that the method had resulted in enlightenment about water use in low income rural communities in Mozambique, to prevent schistosomiasis. However this finding was buried in a mass of unnecessary detail in the preceding sections. Many readers would not get to the key findings as researchers, like myself, are very pushed for time. A good deal of editing is required to shorten this manuscript. 